\title{
Impact of p16 status on pro- and anti-angiogenesis factors in head and neck cancers
}

\author{
P Baruah ${ }^{* 1,2,3}$, M Lee ${ }^{1}$, P O G Wilson ${ }^{4}$, T Odutoye ${ }^{1}$, P Williamson ${ }^{1}, \mathrm{~N} \mathrm{Hyde}^{2}$, J C Kaski ${ }^{3}$ and I E Dumitriu ${ }^{3}$ \\ ${ }^{1}$ Department of ENT, St George's Hospital NHS Trust London, London, UK; ${ }^{2}$ Department of Oral and Maxillo-Facial Surgery, \\ St George's Hospital NHS Trust London, London, UK; ${ }^{3}$ Cardiovascular and Cell Sciences Research Institute, St George's University \\ of London, London, UK and ${ }^{4}$ Department of Pathology, St George's Hospital NHS Trust London, London, UK
}

Background: Head and neck cancers (HNC) are aggressive tumours. Overexpression of p16 in HNC correlates with human papilloma virus (HPV)-associated HNC that carry a better prognosis than HPV-negative tumours. Angiogenesis is an important factor in tumour progression. Our aim was to dissect the impact of p16 expression on angiogenesis factors in HNC.

Methods: Eighteen newly diagnosed HNC patients and controls were analysed. Eleven pro- and anti-angiogenesis factors were quantified using multiplex ELISA in HNC patients and controls. Angiogenesis factors were analysed in tumour tissue using immunohistochemistry.

Results: Circulating levels of endostatin (anti-angiogenesis factor) were higher in the HNC group compared with healthy donors. Interestingly, the pro-angiogenesis factors angiopoietin-1 and vascular endothelial growth factor (VEGF) were significantly higher in patients with p16-negative compared with p16-positive HNC. Moreover, the major source of VEGF in p16-positive HNC tissue was tumour stromal cells. In contrast, both tumour cells and stromal cells expressed VEGF in p16-negative tissue.

Conclusions: We show that p16-negative tumours associate with increased circulating levels of pro-angiogenic VEGF and angiopoietin-1. Tissue expression of VEGF differs between p16-positive and p16-negative tumours. These findings may explain differences in the biological behaviour of p16-positive and p16-negative HNC. Better understanding of mechanisms by which the p16 status influences tumour angiogenesis may guide the development of targeted therapies.

Head and neck cancer (HNC) constitutes an important proportion of cancers worldwide. These are aggressive tumours, and survival rates have remained poor in spite of advances in surgical techniques, radiotherapy and chemotherapy. Interestingly, certain subtypes of HNC are associated with a better prognosis. Sixty per cent of oropharyngeal cancers associate with the oncogenic human papilloma virus (HPV; subtypes 16 and 18; Venuti et al, 2004). Of note, this cohort of patients has a better disease-free survival than the patients who do not express the viral protein (Ang et al, 2010). The reasons underlying this are not known, and further understanding of the biology of these tumours is necessary in order to develop new therapeutic options.
It is well known that tumour growth depends on the establishment of a robust vascular supply to support the oxygen and nutrient requirements of cancer cells. This formation of blood vessel network or angiogenesis also acts as a conduit to allow the spread of cancer cells to the rest of the body (metastases) and thereby promote aggressive tumour progression (Folkman et al, 1989; Hanahan and Folkman, 1996). Vessel turnover in healthy tissue is maintained in the steady state by interactions between molecules that promote angiogenesis (pro-angiogenesis factors) and those that inhibit it (anti-angiogenesis factors; Hanahan and Weinberg, 2011). Tumours exploit this phenomenon and can produce a wide variety of pro-angiogenesis factors to promote their 
own growth, and the levels of these factors in the tumour microenvironment could determine the outcome of cancer therapy. Indeed, anti-angiogenesis therapies in the form of antibodies (for example, anti-vascular endothelial growth factor (VEGF) antibody) or recombinant proteins (for example, endostatin) are being actively used in the treatment of solid tumours (Cohen et al, 2009; Ye et al, 2014).

The aim of this work was to dissect the impact of prognostic factors such as p16, tumour size and nodal status on the angiogenesis profile of HNC. To this purpose, we studied the circulating levels of a panel of 11 angiogenesis factors in patients with HNC and correlated the levels with known prognostic factors including the p16 status. In addition, we explored the effect of the p16 status on the cellular source of angiogenesis factors in the tumour tissue.

\section{MATERIALS AND METHODS}

Study population. Blood samples were obtained from 18 patients with newly diagnosed HNC and 12 healthy controls. The demographic details of patients and controls are provided in Supplementary Table 1. Strict exclusion criteria were applied to reduce the impact of other inflammatory conditions on the serum levels of angiogenesis factors. Patients with co-existing inflammatory disorders such as autoimmune diseases, diabetes, renal failure and cardiac disease were excluded from the study. The study was approved by the local research ethics committee and informed consent was obtained from all study subjects.

Serum isolation and storage. Blood was allowed to coagulate for at least $30 \mathrm{~min}$ at room temperature and then centrifuged at $1500 \mathrm{~g}$ for $10 \mathrm{~min}$ and the serum was stored at a temperature below $-20{ }^{\circ} \mathrm{C}$ until analysis. In one $\mathrm{HNC}$ patient, plasma was used instead of serum.

Multiplex analysis of cytokines. The multiplex Luminex Performance angiogenesis panel was purchased from R\&D Systems (Abingdon, UK). The quantification of cytokines was performed according to the manufacturer's instructions using a Luminex analyser. The following cytokines were analysed: VEGF, VEGF-D, angiopoietin-1, angiogenin, thrombospondin-2, endostatin, placental growth factor (PlGF), platelet-derived growth factor-aa (PDGF-aa), platelet-derived growth factor-bb (PDGF-bb), fibroblast growth factor-acidic (FGF-a) and fibroblast growth factor-basic (FGF-b). Angiopoietin-1 and VEGF could not be quantified in the plasma and therefore one HNC patient was excluded from those analyses.

Immunohistochemistry. The following primary antibodies were used: rabbit polyclonal VEGF (A-20, Santa Cruz Biotechnology, Heidelberg, Germany); goat polyclonal Angiopoietin-1 (R\&D Systems); and goat polyclonal Endostatin (R\&D Systems). Paraffin sections of tumour tissue were cut at $4 \mu \mathrm{m}$ and heated for $45 \mathrm{~min}$ at $60{ }^{\circ} \mathrm{C}$ before staining. Heat antigen retrieval was carried out using Epitope Retrieval Solution 1 (VEGF and endostatin), $\mathrm{pH} 6$ or Epitope Retrieval Solution 2 (Angiopoietin-1), pH 9 at $100^{\circ} \mathrm{C}$ for 20 or $30 \mathrm{~min}$, according to the antibody. Antibodies were diluted $1: 50$ and incubated for $15 \mathrm{~min}$. Negative controls used antibody diluent in place of primary antibody. Visualisation for rabbit polyclonal antibodies was carried out using the Bond Polymer Refine kit (Leica Microsystems, Milton Keynes, UK), an HRPconjugated 3,3'-diaminobenzidine (DAB) detection system, supplied by Leica Microsystems. Visualisation for goat polyclonal antibodies was carried out using the Bond Intense R kit, a Biotin/ streptavidin HRP-conjugated DAB detection system, supplied by Leica Microsystems and secondary antibody-biotinylated rabbit anti-goat, supplied by Dako UK Ltd (Ely, UK). All immunohistochemistry staining was carried out using Bond III Fully automated staining system and associated reagents, supplied by Leica Microsystems. Images were captured using an Olympus BX50 microscope with UPlan Appo lenses and equipped with an Olympus digital camera.

Statistical analysis. Data were compared using the Student's $t$-test. Probability values $(P)$ of less than 0.05 were considered statistically significant. Statistical analysis was performed using the GraphPad Prism software version 5.02, Inc (GraphPad Software, Inc., La Jolla, CA, USA).

\section{RESULTS}

Angiopoietin-1 is higher in the circulation of patients with p16-negative HNC. Angiopoietins are a well-described family of vascular growth factors that have a role in embryonic and tumour angiogenesis (Suri et al, 1996; Holash et al, 1999). We examined the levels of the pro-angiogenesis factor angiopoietin-1 in the serum of HNC $(n=17)$ patients and healthy individuals $(n=13$; Figure 1). No significant differences were observed in the levels of angiopoietin-1 when the total patient cohort was compared with healthy individuals. However, further analysis revealed that angiopoietin-1 levels in the circulation of patients with p16negative tumours were significantly higher compared with those present in healthy individuals (Figure 1B). In contrast, serum angiopoietin-1 levels in patients with p16-positive tumours did not differ from healthy individuals (Figure 1B). We also examined whether the tumour size or nodal status had any impact on the circulating angiopoietin-1 level, neither of which was found to have any influence (Figure $1 \mathrm{C}$ and D).

Vascular endothelial growth factor is higher in the circulation of patients with p16-negative HNC. Vascular endothelial growth factor is upregulated in several tumours and is a potent proangiogenesis factor (Ferrara and Davis-Smyth, 1997; McMahon, 2000). We next evaluated the levels of circulating VEGF in patients with $\mathrm{HNC}(n=17)$ and healthy individuals $(n=13)$. No differences in serum levels of VEGF were observed between the total patient cohort and the healthy controls (Figure 2A). However, in line with our observation with angiopoietin-1, the levels of VEGF were significantly increased in patients with p16-negative tumours compared with healthy individuals (Figure 2B). Moreover, circulating VEGF levels in patients with p16-positive tumours were comparable to those present in healthy individuals (Figure 2B). In contrast to the p16 status, tumour and nodal stage had no influence on circulating VEGF levels (data not shown). Furthermore, whereas circulating levels of VEGF (total) showed a difference between patients carrying p16-positive or -negative tumours, no such difference was observed in VEGF-D levels (Figure 2C and D).

Endostatin levels are higher in patients with HNC compared with controls. We next examined the levels of circulating endostatin in patients with HNC $(n=18)$ and healthy individuals $(n=13)$. Endostatin is a fragment of collagen 18 and is a potent anti-angiogenesis agent (O'Reilly et al, 1997). Endostatin was found to be significantly higher in the serum of patients with HNC compared with healthy individuals (Figure 3A). Unlike VEGF and angiopoietin-1, a significant increase in endostatin was present in both p16-negative and p16-positive HNC patients (Figure 3B). In addition, tumour stage and nodal stage had no influence on endostatin levels (not shown).

Levels of circulating thrombospondin-2, FGF-b, PDGF-aa, PDGF-bb and PIGF in patients with p16-negative and p16-positive HNC. In addition to the previously described angiogenesis factors, we examined the levels of a panel of other angiogenesis factors: thrombospondin-2, PlGF, PDGF-aa and 

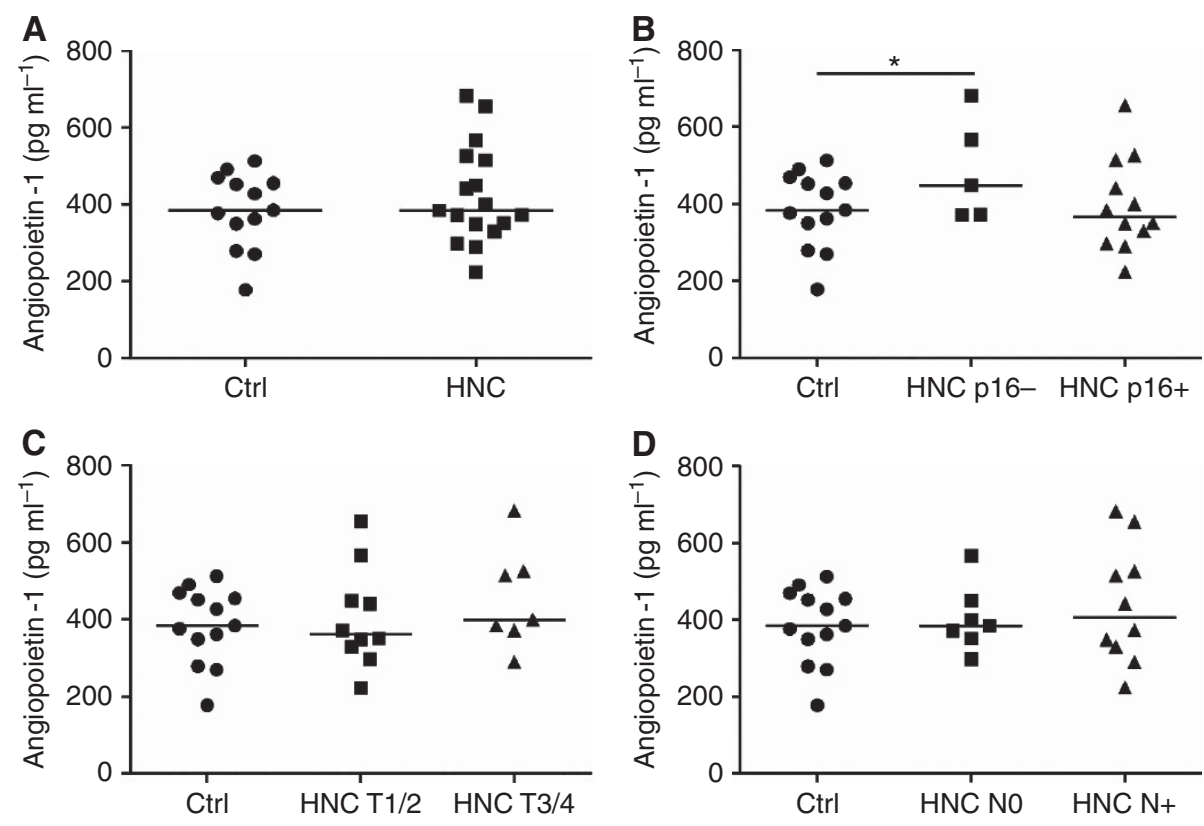

Figure 1. Angiopoietin-1 levels in patients with HNC and controls. Circulating levels of angiopoietin-1 were quantified using multiplex ELISA in sera from healthy individuals $(\mathrm{Ctrl}, n=13)$ and patients with $\mathrm{HNC}(n=17)$. (A) The scatter plots show angiopoietin-1 concentration in the two study groups (horizontal bars show the mean value for each group). (B) Angiopoietin-1 levels were compared between p16-positive (HNC p16 +) or p16negative (HNC p16-) HNC patients and controls. ${ }^{\star} P<0.05$. (C) The plots display angiopoietin-1 in patients with early tumour stage (T1/2), advanced tumour stage (T3/4) and controls. (D) The scatter plots display angiopoietin-1 in patients without nodal disease (N0), nodal disease (N+) and controls.
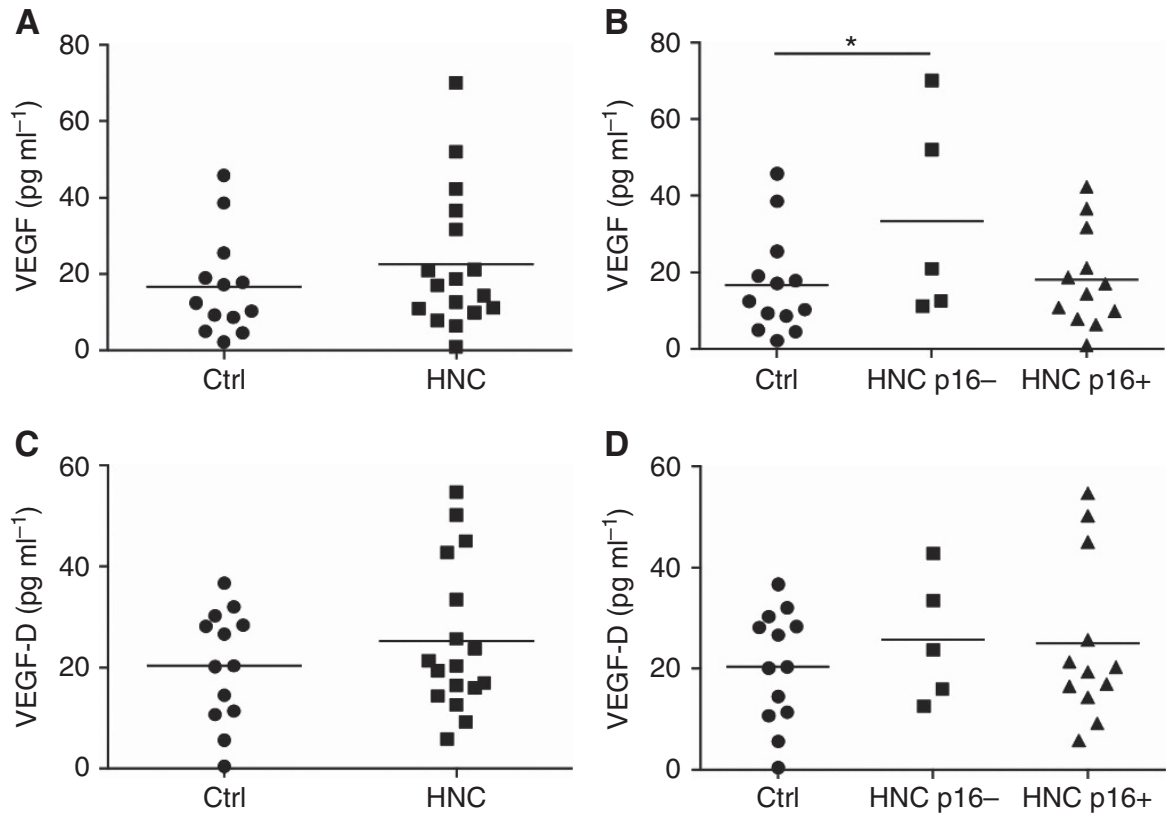

Figure 2. VEGF levels in patients with HNC and controls. Circulating levels of VEGF were quantified using multiplex ELISA in sera from healthy individuals (Ctrl, $n=13)$ and patients with HNC $(n=17)$. (A) The scatter plots show VEGF concentration in the two study groups (horizontal bars show the mean value for each group). (B) VEGF levels were compared between p16-positive (HNC p16 +) or p16-negative (HNC p16-) HNC patients and controls. ${ }^{*} P<0.05$. (C) The plots display VEGF-D in the two study groups. (D) The scatter plots display VEGF-D in p16-positive (HNC $\mathrm{p} 16+)$ and negative $(\mathrm{HNC} \mathrm{p} 16-)$ patients and controls. VEGF $=$ vascular endothelial growth factor.

PDGF-bb, FGF-b and angiogenin. Thrombospondin-2 is an anti-angiogenesis factor (Iruela-Arispe et al, 2004). Whereas thrombospondin-2 levels in the whole patient cohort $(n=18)$ did not differ from controls $(n=13$; Figure $4 \mathrm{~A})$, its levels were found to be lower in sera from patients with p16-positive tumours in comparison with the levels detected in healthy individuals or patients with p16-negative tumours (Figure 4B). This, however, did not reach statistical significance. Patients with T1/2 tumours had lower levels of thrombospondin-2 compared with patients with T3/4 tumours and healthy donors (Figure 4C), but again this did not reach statistical significance. Serum levels of thrombospondin-2 were comparable in patients to $\mathrm{N} 0$ or node-positive disease 

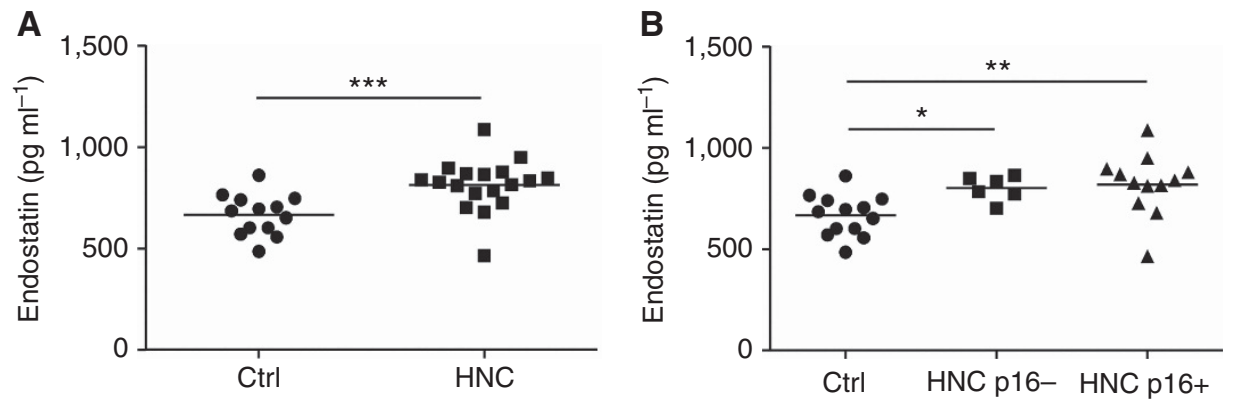

Figure 3. Endostatin levels in patients with HNC and controls. Circulating levels of endostatin were quantified using multiplex ELISA in sera from healthy individuals (Ctrl, $n=13)$ and patients with $\mathrm{HNC}(n=18)$. (A) The scatter plots show endostatin concentration in the two study groups (horizontal bars show the mean value for each group). (B) Endostatin levels were compared between p16-positive (HNC p16+) or p16-negative (HNC p16-) HNC patients and controls. ${ }^{\star \star \star} P<0.001,{ }^{\star \star} P<0.01,{ }^{\star} P<0.05$
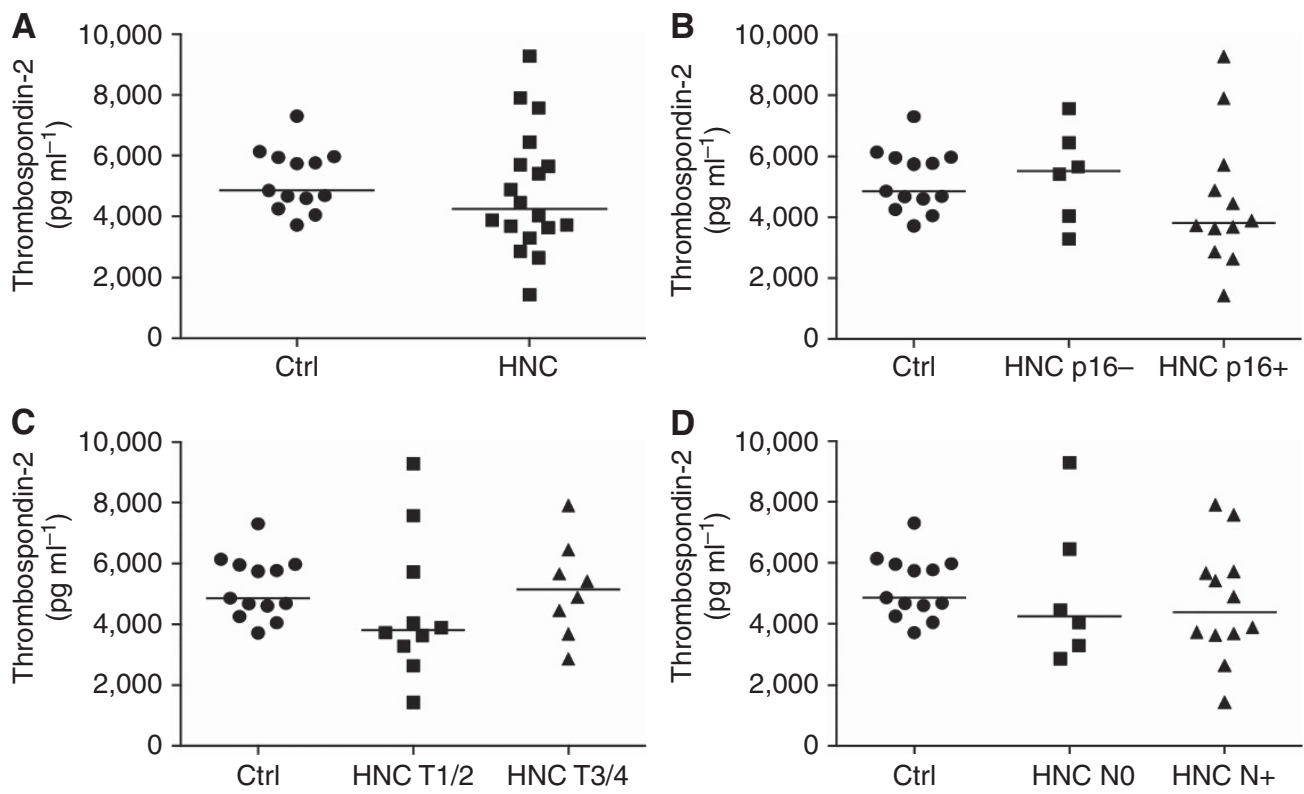

Figure 4. Thrombospondin levels in patients with HNC and controls. Circulating levels of thrombospondin-2 were quantified using multiplex ELISA in sera from healthy individuals (Ctrl, $n=13)$ and patients with $\mathrm{HNC}(n=18)$. (A) The scatter plots show thrombospondin-2 concentration in the two study groups (horizontal bars show the mean value for each group). (B) Thrombospondin-2 levels were compared between p16-positive (HNC p16 +) or p16-negative (HNC p16 - ) HNC patients and controls. (C) The plots display thrombospondin-2 in patients with early tumour stage (T1/2), advanced tumour stage (T3/4) and controls. (D) The scatter plots display thrombospondin-2 in patients without nodal disease (N0), nodal disease $(\mathrm{N}+)$ and controls.

(Figure 4D). Levels of FGF-b, PDGF-aa, PDGF-bb and PlGF were similar in HNC patients compared with the control group (Figure 5A, C, E and G). In addition, the p16 status did not have any influence on the levels of these angiogenesis factors (Figure 5B, $\mathrm{D}, \mathrm{F}$ and $\mathrm{H}$ ). No differences were observed for angiogenin and FGF-a (data not shown). A summary of all angiogenic factors is included in Supplementary Table 2.

Tissue expression of VEGF differs between p16-positive and p16-negative tumours. We next examined the expression of angiopoietin-1, VEGF and endostatin in the HNC tumour tissue using immunohistochemistry. Angiopoietin-1 was localised in tumour tissue to both tumour cells and stromal cells (Figure 6A). This pattern of expression of angiopoietin-1 was similar in both p16-positive and p16-negative tumours. Strong VEGF expression was noted in tumour cells and stromal cells in tissue specimens from p16-negative tumours (Figure 6B left panel). In contrast, expression of VEGF in p16-positive tissue was primarily localised to stromal cells (Figure 6B right panel). Tumour cells in p16positive HNC tissue did not express VEGF in four out of five tissue samples examined. We also examined endostatin in the tumour tissue. Endostatin was expressed in stromal cells resembling macrophages, whereas tumour cells in HNC did not express endostatin. No differences were observed in the tissue expression of endostatin in p16-positive or p16-negative tumour tissue.

\section{DISCUSSION}

This study explored the impact of the p16 status on the profile of angiogenesis factors in HNC, both in terms of circulating levels of these factors and their expression in the tumour tissue. We show an interesting effect of the p16 status on angiogenesis factors in HNC. First, the pro-angiogenesis factors VEGF and angiopoietin-1 are elevated in the serum of patients with p16-negative tumours compared with healthy individuals, whereas this increase was not observed in patients with p16-positive HNC. Second, we show via immunohistochemistry that VEGF production in p16-positive tumours is mainly derived from stromal cells while in p16-negative tumours VEGF is produced both by tumour cells and stromal cells. 
A

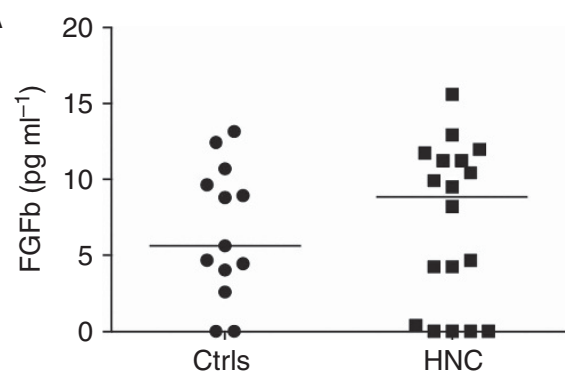

C

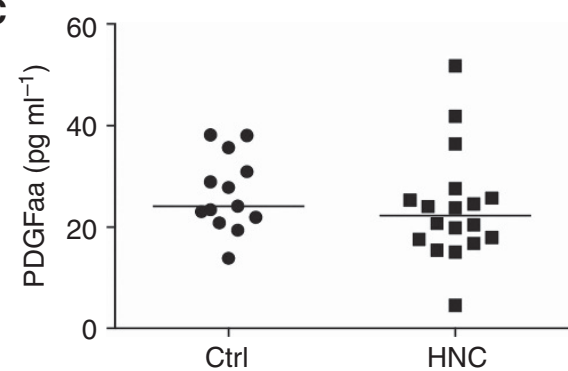

E

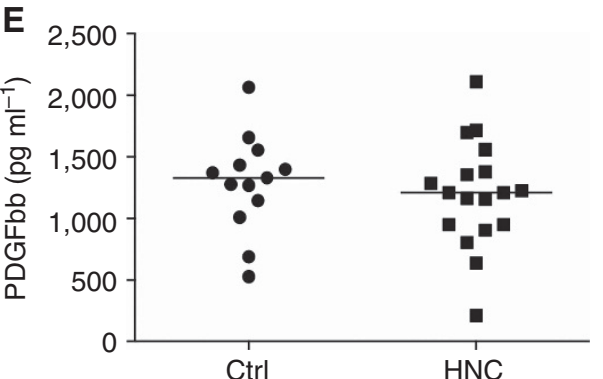

G

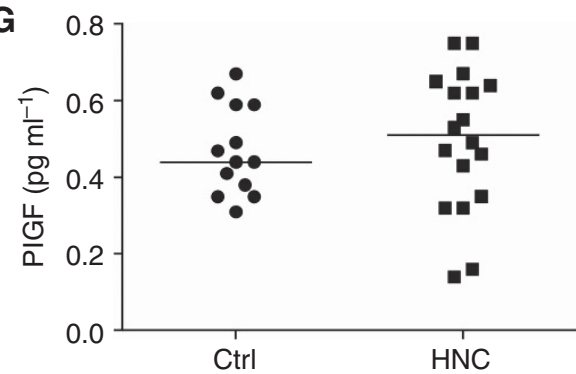

B

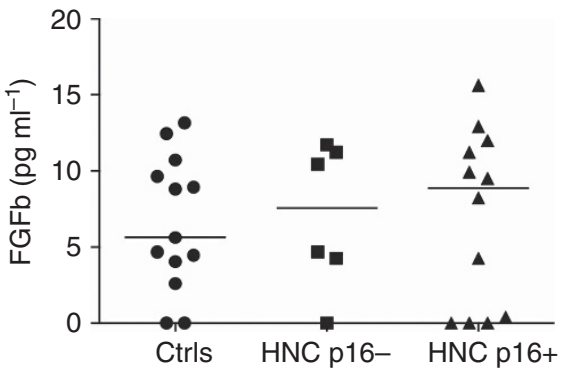

D

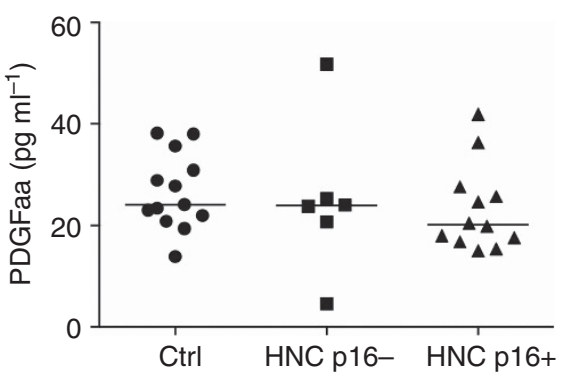

F

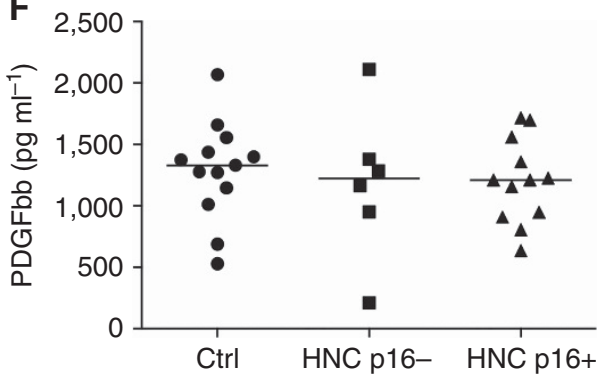

H

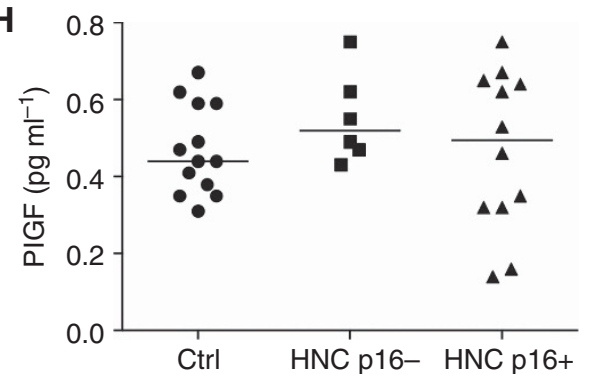

Figure 5. Other angiogenic factor levels in patients with HNC and controls. Circulating levels of angiogenic factors were quantified using multiplex ELISA in sera from healthy individuals (Ctrl, $n=13)$ and patients with HNC $(n=18)$. (A) The scatter plots show FGF-b concentration in controls and HNC patients. Horizontal bars show the mean value for each group. (B) FGF-b levels in p16-positive (HNC p16+) or p16-negative (HNC p16-) HNC patients and controls. (C) The scatter plots show PDGF-aa concentration in controls and HNC patients. (D) PDGF-aa levels in p16-positive or p16-negative HNC patients and controls. (E) The scatter plots show PDGF-bb concentration of controls and HNC patients.

(F) PDGF-bb levels in p16-positive or p16-negative HNC patients and controls. (G) The scatter plots show PIGF concentration controls and HNC patients. (H) PIGF levels in p16-positive or p16-negative HNC patients and controls. FGF-b = fibroblast growth factor-basic; PDGF = plateletderived growth factor; PIGF = placental growth factor.

p16 is considered a good surrogate marker to identify HPVpositive tumours and is widely used in clinical practice. It has been reported that p16 status correlates with a better prognosis in oropharyngeal cancers (Weinberger et al, 2004). In addition, a good correlation between HPV positivity and p16 status has been demonstrated in oropharyngeal cancers (Nichols et al, 2009), which form the majority of our patient cohort (15 of 18 patients). However, detection of p16 by immunohistochemistry carries a false-positive rate of 3-4\% as some HPV-negative tumours could continue to express p16 (Schlecht et al, 2011; Thavaraj et al, 2011; Seiwert, 2013). Although detection of HPV using PCR is the most accurate test for identifying HPV infection, p16 expression using immunohistochemistry continues to be a widely accepted method for HPV detection due to low costs and availability of technical infrastructure in most head and neck units. Of note, ongoing clinical trials such as De-ESCALaTE HPV stratify patients for inclusion on the basis of the HPV status as determined by p16 immunohistochemistry (Masterson et al, 2014). Interestingly, whereas the p16 status does not correlate with HPV positivity in HNC outside the oropharynx such as the oral tongue, it still associates with a better prognosis irrespective of the HPV status (Harris et al, 2011).

It is well established that patients with HPV-positive tumours fare better than patients with HPV-negative tumours (Ang et al, 2010). This has resulted in discussion of altering therapy (deescalation) of HPV-positive tumours by reducing the dose of radiation or trials of cetuximab rather than cisplatin in an effort to reduce the side effects of chemoradiation therapy (Masterson et al, 


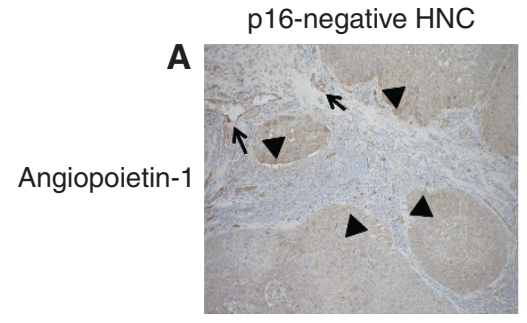

p16-positive HNC
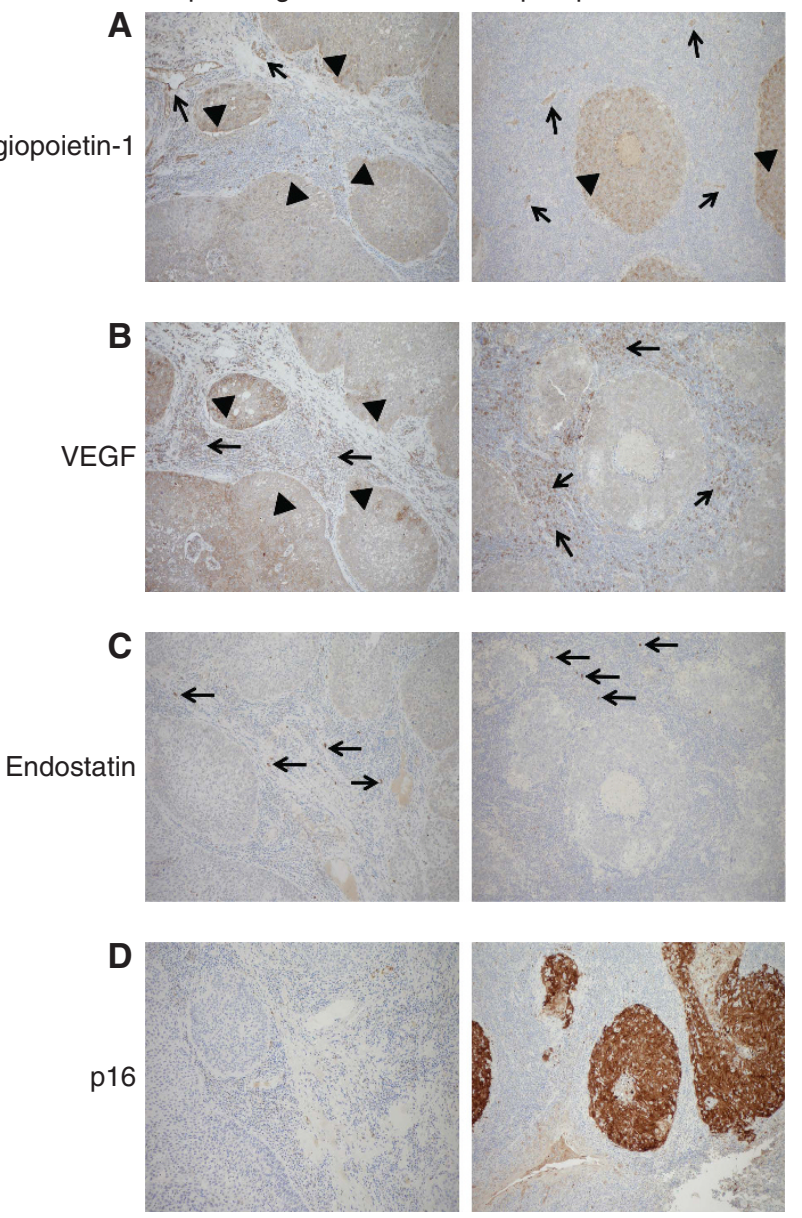

Figure 6. Expression of angiopoietin-1, VEGF and endostatin in p16negative and p16-positive HNC tissue. Expression of angiogenic factors (angiopoietin-1, VEGF and endostatin) was assessed in tissue from five patients with p16-negative and five patients with p16-positive HNC using immunohistochemistry (see Materials and Methods for details). (A) Angiopoietin-1 expression in tumour cells (arrow heads) and tumour stroma (arrows). (B) VEGF expression in tumour tissue (arrow heads) and stromal tissue (arrows). (C) Expression of endostatin in the tumour stroma (arrows). (D) p16 staining in the tumour tissue used for analysis of angiogenesis factor expression.

2014; Tornesello et al, 2014). However, the precise reasons for the different biological behaviours of HPV-positive vs HPV-negative tumours are not certain. Our findings that VEGF and angiopoietin-1 levels are raised in the serum of patients with p16-negative tumours but not those with p16-positive tumours indicates that differences in angiogenic pathways may account, at least in part, for the distinct biological behaviour of p16-positive HNC.

The tumour stroma has emerged as an important contributor to the angiogenesis and progression of cancer (Semenza, 2013). Our immunohistochemistry analysis confirms the important role of the stroma in the production of angiogenesis factors in the HNC microenvironment. Stromal cells such as macrophages are known to produce angiogenesis factors such as VEGF (Lin and Pollard, 2007). We demonstrate that in p16-positive tumour tissue the stromal cells are the predominant source of VEGF, with no expression of VEGF in tumour cells. In contrast, in p16-negative tumours both tumour and stromal cells produce VEGF. This suggests that future work into the mechanisms of VEGF induction in stromal cells vis-a-vis tumour cells may reveal novel strategies to specifically target stromal-derived VEGF. Although our results need to be validated in a larger patient cohort, our findings may have therapeutic potential. Indeed, anti-VEGF antibodies have been used as adjuvants to chemotherapy in the treatment of HNC (Cohen et al, 2009) but are unfortunately associated with potentially fatal complications such as haemorrhage and high blood pressure (Qi et al, 2013; Peng et al, 2014). Inhibitors specific for tumour stromal cell-derived VEGF could allow for inhibition of angiogenesis within p16-positive HNC while leaving normal vasculature intact and avoiding catastrophic side effects.

Our results have also uncovered interesting facets of endostatin, which is an anti-angiogenesis factor that has been shown to be elevated in a variety of cancers (Koc et al, 2006; Kantola et al, 2014). We also found significantly elevated levels of endostatin in our HNC patient cohort, albeit there was no difference between p16-positive and p16-negative tumours. We found that endostatin is expressed in tumour stroma in cells resembling macrophages. To our knowledge, this is the first demonstration of endostatin production within the cancer stromal tissue, which warrants further investigation to define the role of endostatin in the tumour microenvironment. Recombinant endostatin therapy has shown good response in mouse models in early studies (Blezinger et al, 1999), but human tumour response was not encouraging (Thomas et al, 2003), possibly due to a failure to achieve adequate local concentrations of endostatin required to inhibit tumour growth. Endostatin delivery to tumour tissue using viral vectors is currently in trials (Ye et al, 2014).

The p16 status was also not found to have an impact on the production of other angiogenesis factors such as FGF-b, PDGF and PIGF. However, thrombospondin-2 levels showed a trend to be lower in the serum of patients with p16-positive tumours compared with patients with p16-negative tumours and even healthy donors (not statistically significant). High levels of thrombospondin-2 in lung cancer have been correlated to poorer prognosis (Naumnik et al, 2015), whereas downregulation of thrombospondin-2 in gastric cancer correlates with poorer prognosis (Sun et al, 2014). The relationship between thrombospondin-2 and p16 status is not clear currently, but future work could shed light on this.

In summary, alterations in angiogenesis pathways in p16positive vs p16-negative $\mathrm{HNC}$ could be one of the factors underlying the different biological behaviour of these two types of HNC. Future studies in larger patient cohorts and direct demonstration of HPV using PCR techniques within tumour tissue will provide further confirmation of the role of HPV in this process. In addition, the identification of stromal cells as important producers of angiogenesis factors, in particular VEGF and endostatin, opens new avenues for research on targets for selective inhibition or induction of angiogenesis factors from stromal cells in HNC.

\section{ACKNOWLEDGEMENTS}

We are very grateful to all the patients and healthy subjects for their participation in the study. This work was funded by the Royal College of Surgeons of Edinburgh (grant no. SRG/10/033 and SRG/12/059 to PB) and St George's Hospital Charitable Foundation. Work in the Cardiovascular Immunology laboratory at St George's University of London is funded by the British Heart Foundation (grant no. PG/10/50/28434, PG/13/24/30115, $\mathrm{PG} / 14 / 18 / 30724$ to IED).

\section{CONFLICT OF INTEREST}

The authors declare no conflict of interest. 


\section{REFERENCES}

Ang KK, Harris J, Wheeler R, Weber R, Rosenthal DI, Nguyen-Tan PF, Westra WH, Chung CH, Jordan RC, Lu C, Kim H, Axelrod R, Silverman CC, Redmond KP, Gillison ML (2010) Human papillomavirus and survival of patients with oropharyngeal cancer. N Engl J Med 363(1): 24-35.

Blezinger P, Wang J, Gondo M, Quezada A, Mehrens D, French M, Singhal A, Sullivan S, Rolland A, Ralston R, Min W (1999) Systemic inhibition of tumor growth and tumor metastases by intramuscular administration of the endostatin gene. Nat Biotechnol 17(4): 343-348.

Cohen EE, Davis DW, Karrison TG, Seiwert TY, Wong SJ, Nattam S, Kozloff MF, Clark JI, Yan DH, Liu W, Pierce C, Dancey JE, Stenson K, Blair E, Dekker A, Vokes EE (2009) Erlotinib and bevacizumab in patients with recurrent or metastatic squamous-cell carcinoma of the head and neck: a phase I/II study. Lancet Oncol 10(3): 247-257.

Ferrara N, Davis-Smyth T (1997) The biology of vascular endothelial growth factor. Endocr Rev 18(1): 4-25.

Folkman J, Watson K, Ingber D, Hanahan D (1989) Induction of angiogenesis during the transition from hyperplasia to neoplasia. Nature 339(6219): $58-61$.

Hanahan D, Folkman J (1996) Patterns and emerging mechanisms of the angiogenic switch during tumorigenesis. Cell 86(3): 353-364.

Hanahan D, Weinberg RA (2011) Hallmarks of cancer: the next generation. Cell 144(5): 646-674.

Harris SL, Thorne LB, Seaman WT, Hayes DN, Couch ME, Kimple RJ (2011) Association of p16(INK4a) overexpression with improved outcomes in young patients with squamous cell cancers of the oral tongue. Head Neck 33(11): 1622-1627.

Holash J, Maisonpierre PC, Compton D, Boland P, Alexander CR, Zagzag D, Yancopoulos GD, Wiegand SJ (1999) Vessel cooption, regression, and growth in tumors mediated by angiopoietins and VEGF. Science 284(5422): 1994-1998.

Iruela-Arispe ML, Luque A, Lee N (2004) Thrombospondin modules and angiogenesis. Int J Biochem Cell Biol 36(6): 1070-1078.

Kantola T, Vayrynen JP, Klintrup K, Makela J, Karppinen SM, Pihlajaniemi T, Autio-Harmainen H, Karttunen TJ, Makinen MJ, Tuomisto A (2014) Serum endostatin levels are elevated in colorectal cancer and correlate with invasion and systemic inflammatory markers. Br J Cancer 111(8): 1605-1613.

Koc M, Gocmen E, Kilic M, Ozbay M, Oktem M, Tez M (2006) Serum endostatin levels in gastric cancer patients: correlation with clinicopathological parameters. Hepatogastroenterology 53(70): 616-618.

Lin EY, Pollard JW (2007) Tumor-associated macrophages press the angiogenic switch in breast cancer. Cancer Res 67(11): 5064-5066.

Masterson L, Moualed D, Liu ZW, Howard JE, Dwivedi RC, Tysome JR, Benson R, Sterling JC, Sudhoff H, Jani P, Goon PK (2014) De-escalation treatment protocols for human papillomavirus-associated oropharyngeal squamous cell carcinoma: a systematic review and meta-analysis of current clinical trials. Eur J Cancer 50(15): 2636-2648.

McMahon G (2000) VEGF receptor signaling in tumor angiogenesis. Oncologist 5(Suppl 1): 3-10.

Naumnik W, Ossolinska M, Plonska I, Chyczewska E, Niklinski J (2015) Circulating thrombospondin-2 and FGF-2 in patients with advanced non-small cell lung cancer: correlation with survival. Adv Exp Med Biol 833: 9-14.

Nichols AC, Faquin WC, Westra WH, Mroz EA, Begum S, Clark JR, Rocco JW (2009) HPV-16 infection predicts treatment outcome in oropharyngeal squamous cell carcinoma. Otolaryngol Head Neck Surg 140(2): 228-234.

O’Reilly MS, Boehm T, Shing Y, Fukai N, Vasios G, Lane WS, Flynn E, Birkhead JR, Olsen BR, Folkman J (1997) Endostatin: an endogenous inhibitor of angiogenesis and tumor growth. Cell 88(2): 277-285.

Peng L, Bu Z, Zhou Y, Ye X, Liu J, Zhao Q (2014) Hemorrhagic events in cancer patients treated with aflibercept: a meta-analysis. Tumour Biol 35(9): 9419-9427.

Qi WX, Tang LN, Sun YJ, He AN, Lin F, Shen Z, Yao Y (2013) Incidence and risk of hemorrhagic events with vascular endothelial growth factor receptor tyrosine-kinase inhibitors: an up-to-date meta-analysis of 27 randomized controlled trials. Ann Oncol 24(12): 2943-2952.

Schlecht NF, Brandwein-Gensler M, Nuovo GJ, Li M, Dunne A, Kawachi N, Smith RV, Burk RD, Prystowsky MB (2011) A comparison of clinically utilized human papillomavirus detection methods in head and neck cancer. Mod Pathol 24(10): 1295-1305.

Seiwert T (2013) Accurate HPV testing: a requirement for precision medicine for head and neck cancer. Ann Oncol 24(11): 2711-2713.

Semenza GL (2013) Cancer-stromal cell interactions mediated by hypoxiainducible factors promote angiogenesis, lymphangiogenesis, and metastasis. Oncogene 32(35): 4057-4063.

Sun R, Wu J, Chen Y, Lu M, Zhang S, Lu D, Li Y (2014) Down regulation of Thrombospondin 2 predicts poor prognosis in patients with gastric cancer. Mol Cancer 13: 225.

Suri C, Jones PF, Patan S, Bartunkova S, Maisonpierre PC, Davis S, Sato TN, Yancopoulos GD (1996) Requisite role of angiopoietin-1, a ligand for the TIE2 receptor, during embryonic angiogenesis. Cell 87(7): $1171-1180$

Thavaraj S, Stokes A, Guerra E, Bible J, Halligan E, Long A, Okpokam A, Sloan P, Odell E, Robinson M (2011) Evaluation of human papillomavirus testing for squamous cell carcinoma of the tonsil in clinical practice. J Clin Pathol 64(4): 308-312.

Thomas JP, Arzoomanian RZ, Alberti D, Marnocha R, Lee F, Friedl A, Tutsch K, Dresen A, Geiger P, Pluda J, Fogler W, Schiller JH, Wilding G (2003) Phase I pharmacokinetic and pharmacodynamic study of recombinant human endostatin in patients with advanced solid tumors. J Clin Oncol 21(2): 223-231.

Tornesello ML, Perri F, Buonaguro L, Ionna F, Buonaguro FM, Caponigro F (2014) HPV-related oropharyngeal cancers: from pathogenesis to new therapeutic approaches. Cancer Lett 351(2): 198-205.

Venuti A, Badaracco G, Rizzo C, Mafera B, Rahimi S, Vigili M (2004) Presence of HPV in head and neck tumours: high prevalence in tonsillar localization. J Exp Clin Cancer Res 23(4): 561-566.

Weinberger PM, Yu Z, Haffty BG, Kowalski D, Harigopal M, Sasaki C, Rimm DL, Psyrri A (2004) Prognostic significance of p16 protein levels in oropharyngeal squamous cell cancer. Clin Cancer Res 10(17): 5684-5691.

Ye W, Liu R, Pan C, Jiang W, Zhang L, Guan Z, Wu J, Ying X, Li L, Li S, Tan W, Zeng M, Kang T, Liu Q, Thomas GR, Huang M, Deng W, Huang W (2014) Multicenter randomized phase 2 clinical trial of a recombinant human endostatin adenovirus in patients with advanced head and neck carcinoma. Mol Ther 22(6): 1221-1229.

This work is published under the standard license to publish agreement. After 12 months the work will become freely available and the license terms will switch to a Creative Commons AttributionNonCommercial-Share Alike 4.0 Unported License

Supplementary Information accompanies this paper on British Journal of Cancer website (http://www.nature.com/bjc) 